\title{
Laser-Induced Damage Density Measurements of Optical Materials
}

\author{
Laurent Lamaignère \\ CEA, DAM, CESTA, BP N², F-33114 Le Barp, \\ France
}

\section{Introduction}

The prediction of the lifetime of optics in high power fusion lasers is a key point for mastering the facilities (Bercegol et al., 2008). The laser damage scenario is seen as occurring in two distinct steps. The first one concerns the damage occurrence due to the first optic irradiation: the initiation step (Feit et al., 1999). Afterwards, damage sites are likely to grow with successive new shots: the growth step (Norton et al., 2006 ; Negres et al., 2010). The damage growth study requires the use of large beams due to the exponential nature of the process, leading to centimetre damage sites. At the same time, damage density measurements on large optics are mainly performed off-line by raster scanning the whole component with small Gaussian beams ( $\phi \sim 1 \mathrm{~mm})$ (Lamaignère et al., 2007), except for a wide range of results using larger beams which permit also a comparison between procedures (DeMange et al., 2004). Tests are currently done at a given control fluence. The goal is to irradiate a known area in order to reveal all the defects which could create damage. This procedure, named rasterscan procedure, gives access to the optical damage densities (Lamaignère et al., 2007). This measurement is accurate and reproducible (Lamaignère et al., 2010). In standard tests (ISO standards, 2011), results are given in terms of damage probability. This data treatment is size dependent; on the contrary, it is important to focus the attention rather on the density of sites that damage at a given fluence.

The purpose of the present chapter is to explain how the knowledge of the entire test parameters leads to a comparable and reproducible metrology whatever beam and test characteristics. To this end, a specific mathematical treatment is implemented which takes into account beam shape and overlap. This procedure, which leads to very low damage densities with a good accuracy, is then compared with $1 / 1$ tests using small beams. It is also presented with a peculiar attention on data reduction. Indeed an appropriate treatment of these tests results into damage densities gives at once good complementarity of the several procedures and permits the use of one procedure or the other, depending on the need. This procedure is also compared with tests realized with large beams (centimetre sized as used on high power facilities). That permits to compare results given by specific table-top test facilities with those really obtained on laser lines (Lamaignère et al., 2011).

In section 2, facilities using small and large beams are described. The several procedures used are presented in section 3. Developments of data treatment and analysis are given in section 4 . Section 5 is devoted to the determination of error bars on both the fluence 
measurement and the damage density. Few results presented in section 6 illustrate the complementarity of procedures, the repeatability, the reproducibility and the representativeness.

\section{Tests facilities}

\subsection{Small beam facilities}

Measurements are currently performed on Q-switched Nd:YAG lasers supplying three wavelengths, the fundamental $(1.064 \mu \mathrm{m}, 1 \omega)$, the second $(0.532 \mu \mathrm{m}, 2 \omega)$ and third $(0.355 \mu \mathrm{m}$, $3 \omega)$ harmonics. Laser injection seeding ensures a longitudinal monomode beam and a stable temporal profile. The lasers deliver approximately $1 \mathrm{~J}$ at a nominal repetition rate of $10 \mathrm{~Hz}$ at $1 \omega$. Beam is focused into the sample by a convex lens which focal length is few meters. It induces a depth of focus (DOF) bigger than the sample thickness, ensuring the beam shape to be constant along the DOF.

Two parameters are essential in damage tests: the beam profile and the energy of each shot. Equivalent surface $S_{e q}$ (i.e. defined as the surface given at 1/e for a Gaussian beam) of the beam is determined at the equivalent plane corresponding to distance between the focusing lens and the sample (see Fig. 1). At the focus point, beam is millimetric Gaussian shaped and diameter (given at 1/e) is around 0.5 to $1 \mathrm{~mm}$. Shot-to-shot laser fluences fluctuations (about $15 \%$ at one standard deviation) are mainly due to fluctuations of the equivalent surface of the beam. This is the reason why fluence has to be determined for each shot:

- $\quad$ Energy measurements are sampled by pyroelectric cell at $10 \mathrm{~Hz}$ : it is a relative data; this cell has to be systematically compared with full-beam calorimeter calibrations before each test.

- A photoelectric cell records the temporal profile (rise time of about $70 \mathrm{ps}$ ). This parameter is rather stable (standard deviation of less than $2 \%$ of the average value). Nevertheless, it is important to check that the laser goes on working with a single longitudinal mode.

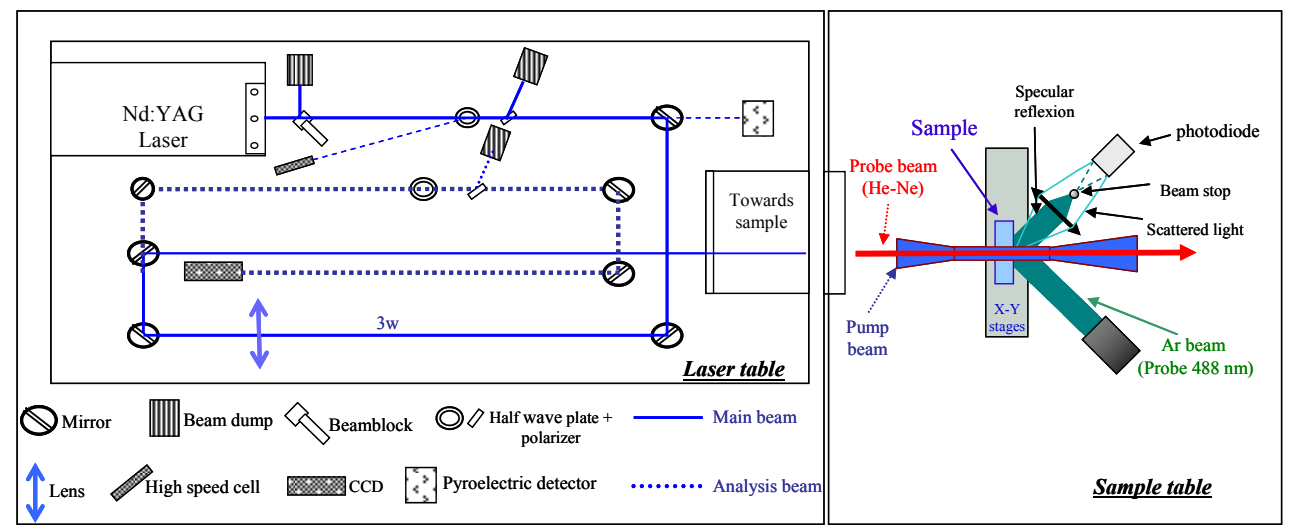

Fig. 1. Typical experimental setup for laser damage testing (Nd:Yag facility) with small beam. 
- A CCD camera records the spatial profile. This tool is positioned at a position optically equivalent to that of the sample. The two main parameters obtained from this set-up are the maximum value (pixel) of the beam which is directly connected to the maximum fluence, and the pointing position which permits to build up the fluence map. Beam wings integrate a large fraction of the energy (up to 50\%) and are responsible for a large part of the fluctuations. Above a diameter of $1 / \mathrm{e}$, the shape is very nearly Gaussian.

\subsection{Large beam facilities}

The advantage of tests with large beams is that they are representative of real shots on high power lasers. They are carried out on high power facilities where parametric studies are conducted: pulse duration or phase modulation (FM) effects. With energies about 100J at $1053 \mathrm{~nm}$ and 50J at 351nm and after size reduction of the beam on the sample, high fluences are delivered with beam diameters about $16 \mathrm{~mm}$. Due to a contrast inside the beam itself (peak to average) of about few units, a shot at a given average fluence covers a large range of local fluences (Fig. 2). The laser front-end capability makes possible parametric studies like the effect of FM, temporal shapes and pulse durations on laser damage phenomenology. The characteristics of this kind of laser are quite similar to high-power lasers for fusion research: the front-end, the amplification stage, the spatial filters, the frequency converters crystals. Then laser damage measurements performed with this system should be representative of the damage phenomenon on high-power lasers.
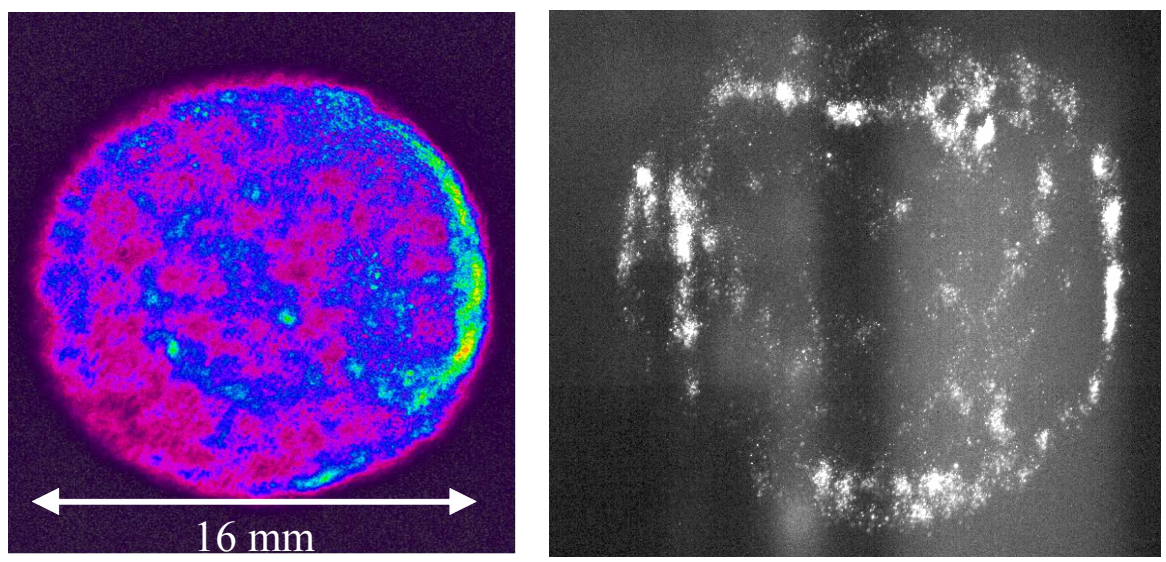

Fig. 2. Damage test with a centimetre-sized beam. On the left, spatial profile of the $16 \mathrm{~mm}-$ diametre beam at the sample plane as measured on CCD camera. On the right, the corresponding damage photography is reported. Matching the two maps allows to extract the fluence for each damage site.

\section{Procedures}

\subsection{1on1 procedure}

The $1 / 1$ test is made on a limited number of sites (ISO standards, 2011). Results are generally given in terms of damage probability as a function of fluence. Since the beam sizes 
of several benches are different, it is compulsory to convert probability into damage density. $1 / 1$ tests are also done because with a small number of tested sites, a rapid result is achieved. Another advantage is that a relative comparison of several optical components is possible if the test parameters are unchanged. Generally, the $1 / 1$ test is used instead of $S / 1$ to avoid material ageing or conditioning effects.

\subsubsection{Small beam}

In the $1 / 1$ procedure:

- 1 shot is fired on 1 pristine site. Energy, spatial and temporal profiles are recorded and lead to beam fluence and intensity for each shot.

- Damage detection is achieved by means of a probe laser beam co-linear to the pump laser beam.

- Many shots are realized at different fluences (about twenty sites are tested per fluence, on the basis of a ten of fluences set); gathering shots by fluence group, probability plots versus fluence are determined.

The next step is to convert the probability into damage density. This data treatment is presented on paragraph 4.1 .

\subsubsection{Large beam}

The metrology is very close to small beam metrology: energy, temporal and spatial profiles are recorded. For the latest, a CCD camera is positioned at a plane optically equivalent to that of the sample. Beam profile and absolute energy measurement give access to energy density $F_{(x, y)}$ locally in the beam:

$$
F(x, y)=\frac{E_{t o t}}{S_{p i x} \times \sum_{i=\min }^{i=\max }\left(n_{i} \times i\right)} \times i(x, y)
$$

$E_{\text {tot }}:$ total energy

$S_{\text {pix }}:$ CCD pixel area

$i_{(x, y)}$ : pixel grey level

Figure 2 shows fluence spatial distribution of a large beam shot (beam diameter of about 16 $\mathrm{mm}$ ). Due to a beam contrast (peak to average) of about $40 \%$, a shot at a requested fluence (mean value) covers a large range of fluences. This make compulsory the exact correlation between local fluence and local event (damage or not). Data treatment is then completed by matching fluence and damage maps (map superposition is realized by means of reference points in the beam, hot spots). The two maps are compared pixel to pixel to connect a local fluence with each damage site detected. Then data are arranged to determine a damage density for each $1 \mathrm{~J} / \mathrm{cm}^{2}$ wide class of fluence. Damage density versus fluence is then given by the following relation:

$$
\mathrm{D}(\mathrm{f})=-\ln (1-\mathrm{P}) / \mathrm{S}
$$

Where $P$ is the damage probability: rate between the damage sites number $\left(N_{d}\right)$ over the irradiated sites number $\left(N_{t}\right)$ and $S$ is the size of the pixel camera. 


\subsection{Rasterscan procedure}

In order to scan a large area, the sample to be tested is translated continuously along a first direction and stepped along a second direction (Fig.3), the laser working at predetermined control fluence. Repeating this test at several fluences on different areas permits to determine the number of damage sites versus fluence thus the damage density. But in the case of Nd:Yag tripled frequency, shot to shot fluence fluctuations have to be taken into account. Fluence can display a standard deviation of up to $15 \%$ for this kind of laser (Fig. 5 of reference (Lamaignère et al., 2007)). When thousands of shots are fired, modulation of a factor 2 in fluence is obtained. Thus, it is important to monitor the specific fluence of each shot in order to get a precise correlation between damage occurrence and laser fluence. During the test, energy, spatial and temporal profiles, beam position on the sample are recorded for each shot (at $10 \mathrm{~Hz}$ ) in order to build up an accurate map of peak fluences corresponding to the scan.

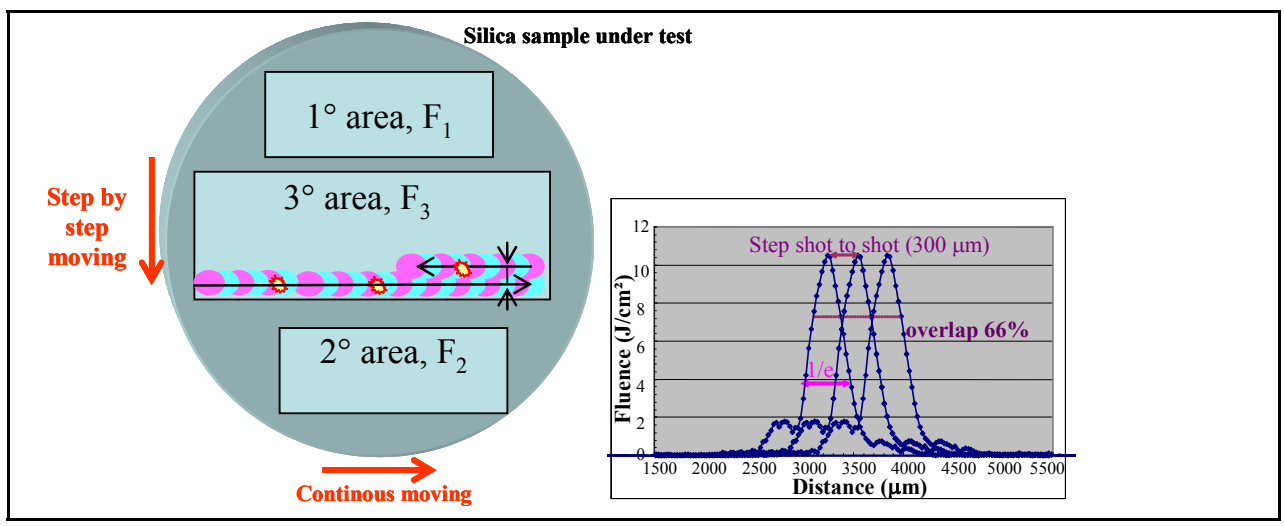

Fig. 3. On the left, the successive laser pulses overlap spatially to achieve an uniform scanning. On the right, the beam overlap.

Much attention has to be paid to the position of the zero level in the beam image, because the value of fluence is very sensitive to small errors in that level. The determination of that position has to be checked very often, for example by verifying that the total energy integrated on the CCD is proportional to the pyroelectric cell measurement. The uncertainty on the zero level is responsible for the largest part of the total uncertainty on fluence measurement. This point is treated on paragraph 5.1.

\subsection{Damage detection system}

Depending on facilities, damage detection is realized in-situ after each shot during scans or after the irradiation with a "postmortem" observation. The "post-mortem" observation of irradiated areas is realized by means of a long working microscope (Fig. 6 of ref. (Lamaignère et al., 2007)). The minimum damage size detected is about $10 \mu \mathrm{m}$ whatever the morphology of damage. Below this value, it is difficult to discriminate between a damage site and a defect of the optic that did not evolve due to the shots. Damage sites might be of different types: some are rather deep with fractures, others are shallower. 
In the case of the in-situ detection, a probe beam is focused at the same position as the pump laser on the sample. The specular reflexion is stopped by a pellet set at the centre of a collecting lens. The probe beam is scattered when damage occurs and the collecting lens redirects the stray light onto a photocell: that corresponds to a Schlieren diagnostic. Since the optic moves during the scan, the signal is recorded just before and just after the shot. The comparison of the two signals allows making a decision on damage occurrence or not. The smallest damage detected is again about $10 \mu \mathrm{m}$.

Matching the maps of fluence and damage sites allows one to extract the peak fluence $F_{p}$ for each damage site. A first data treatment is then realized by gathering damage sites in several fluence groups $\left[\left(\mathrm{F}_{\mathrm{p}}-\Delta \mathrm{F}_{\mathrm{p}} / 2\right)\right.$ to $\left.\left(\mathrm{F}_{\mathrm{p}}+\Delta \mathrm{F}_{\mathrm{p}} / 2\right)\right]$. Knowing damage number and shot number, and attributing an area to each shot, the damage density is determined for each group (Fig. 4). Then with only one predetermined control fluence, damage density is then determined on a large fluence range, due to fluence fluctuations during scan. At this point, however, only the relation between damage sites and peak fluences is available. In next section, damage density is calculated as a function of local fluence.

\section{Data treatment}

\subsection{1/1 procedure}

The damage probability is converted into damage density. Note that interaction between materials defects is neglected. Thus, if defects damaging at a given energy density $F$ are randomly distributed, the defect density $D(F)$ follows a Poisson law. Then, on a given area $S$, damage probability $P(F, S)$ and defect density $D(F)$ are related by (Feit et al., 1999):

$$
P(F, S)=1-\exp (-\mathrm{D}(\mathrm{F}) \cdot S)
$$

The treatment is the same considering surface or volume damage densities. The total volume illuminated $V$ is the product of the beam area by the optical component thickness $\theta$ (Lamaignère al al., 2009). $V_{\text {eq.g }}$ is defined as the Gaussian beam equivalent volume $\left(S_{\text {eq.g }}\right.$ being the Gaussian beam equivalent area):

$$
V_{e q \cdot g}=S_{e q \cdot g} \cdot \theta
$$

Defining $\delta_{m}$ as the measured damage density which is then obtained from damage probability:

$$
P=1-\exp \left(-\delta_{m} \cdot S_{\text {eq.g }}\right)
$$

The measured damage density is then:

$$
\delta_{m}=-\frac{\ln (1-P)}{S_{e q \cdot g}}
$$

When a spatially Gaussian beam is used, the absolute damage density can be expressed as the logarithmic derivative of the measured density $\delta_{m}$ : 


$$
D(F)=F \cdot \frac{d \delta_{m}(F)}{d F}
$$

The experimental curve of $\delta_{m}(F)$ can often be fitted by a power law of the energy density:

$$
\delta_{m}(F)=\alpha \cdot(F)^{\beta}
$$

Where $\alpha$ and $\beta$ are calculated from the best fit of the measurements.

From equations (7) and (8), the absolute damage density is obtained:

$$
D(F)=\beta . \delta_{m}(F)
$$

Or again:

$$
D(F)=\beta \cdot\left(-\frac{\ln (1-P)}{S_{e q . g}}\right)
$$

Note that if $\mathrm{P}<<1$, then relation (10) is equivalent to:

$$
D(F)=\beta \cdot \frac{P}{S_{\text {eq.g }}}
$$

For each energy density level $F$, the observed damage probability $P$ is given by the equation:

$$
P=\frac{n^{D}}{n^{D}+n^{N D}}
$$

where $\left(n^{D}+n^{N D}\right)$ is the total number of exposed sites and $n^{D}$ the number of damage sites. Then, if $n^{D<<} n^{D}+n^{N D}$, the measured damage density is:

$$
\delta_{m}(F)=\frac{n^{D}}{\left(n^{D}+n^{N D}\right) \cdot S_{e q \cdot g}}
$$

Finally, the absolute damage density given in (11) is:

$$
D(F)=\beta \cdot \frac{n^{D}}{\left(n^{D}+n^{N D}\right) \cdot S_{e q \cdot g}}
$$

In order to determine the absolute damage density, the knowledge of the number of damage sites and the Gaussian beam equivalent area is sufficient. The final step consists in determining the exponent $\beta$ from power law fitting of experimental data.

For a top-hat beam, the absolute damage density is directly given by equation (13), the same equations can be used than for Gaussian beams taking $\beta=1$

\subsection{Rasterscan procedure}

In this part, the calculations used to analyze the experimental data are described. When tests are done with small quasi-Gaussian beams, the overlap is not perfect (see the right insert 
figure 3): a large area is irradiated at a fluence smaller than peak fluence. Then the precise beam shape and more precisely the Gaussian equivalent area have to be taken into account. Each shot is identified with its maximum fluence $F_{p}$. On the high fluence side, it is observed experimentally that the damage density varied rapidly with fluence, approximately as a power law (Fig. 4). The shape of the beam at the top is then the relevant function. Shots are thus characterized by the equivalent area of the Gaussian peak. When this is done, it appears that, schematically, the resulting curve could be divided in two parts (Fig. 4).

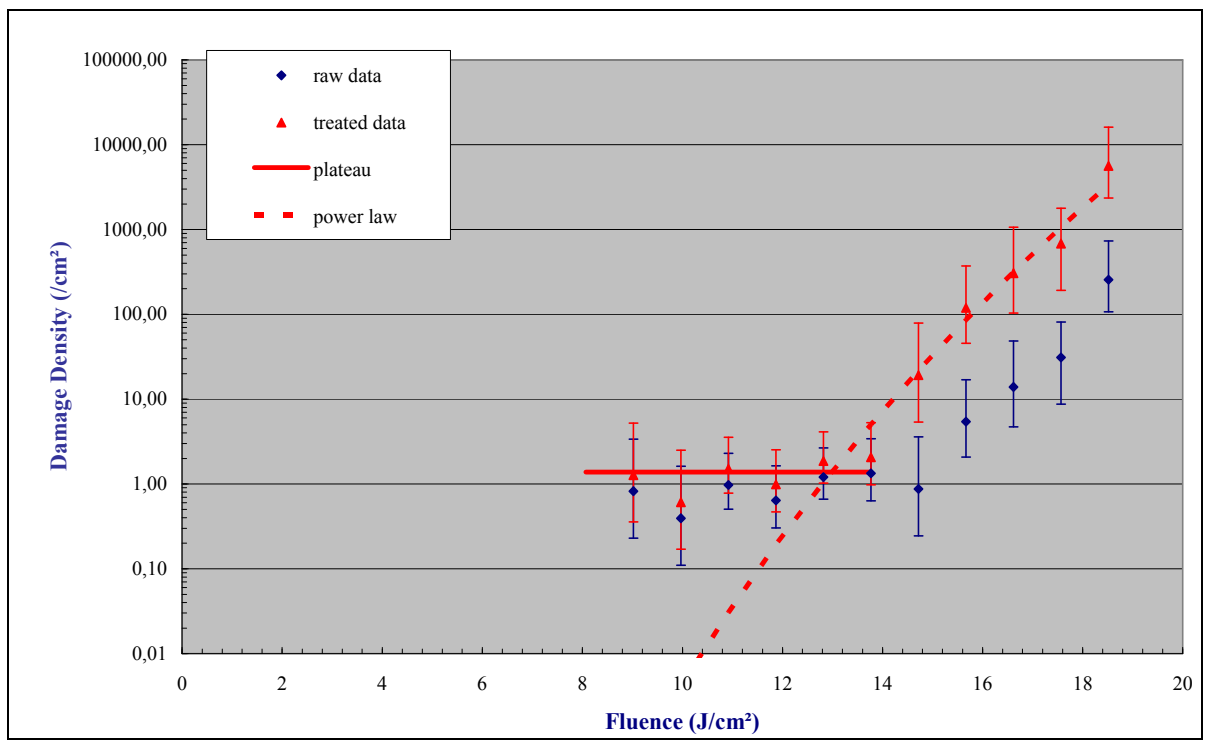

Fig. 4. Damage density versus fluence, after treatment taking care of beam shape and to derive experimental uncertainty. Diamonds are the raw data. Triangles represent treated data; in this case fluence is the local fluence. Data on plateau are issue from relation (23) and those in the high fluence range from relation (22). Error bars calculations are explained in §5.2.

\subsubsection{At high fluences, above $F_{\text {cut }}$, (see Fig. 4)}

Damage density increases quickly with fluence. Calculations in this section are made easy by considering spatially Gaussian shapes, for which fluence distribution can be expressed as a function of $r$, the distance to the peak. Each shot is identified with its peak fluence $F_{p}$.

$$
F(r)=F_{p} \exp \left(-\frac{\pi r^{2}}{S_{\text {eq.g }}}\right)
$$

becomes, when derived

$$
\frac{d F}{F}=\frac{2 \pi r d r}{S_{\text {eq.g }}}
$$

where we defined: 
$S_{\text {eq.g }}=$ Gaussian beam equivalent area.

Let us also define, for the following equations

S(total): Total scanned surface area

$\mathrm{F}_{\text {cut }}$ : Transition fluence between "plateau" and power law behaviors

$\mathrm{N}$ : Number of damage sites

n: Number of shots

Let $\mathrm{D}(\mathrm{F})$ be the damage density distribution that we are trying to measure, a function of local fluence $\mathrm{F}$. We call $\delta_{\mathrm{m}}\left(\mathrm{F}_{\mathrm{p}}\right)$ the experimentally measured density at $\mathrm{F}_{\mathrm{p}}$. The number of damage sites created by a shot of maximum fluence $F_{p}$ for a Gaussian beam is given by the relation:

$$
N\left(F_{p}\right)=\int_{0}^{+\infty} 2 \pi r \cdot d r \cdot D[F(r)]=\int_{0}^{F_{p}} S_{e q \cdot g} \cdot d F \cdot \frac{D[F]}{F}
$$

The third member of eq. (17) is obtained by changing variables in the integral and using eq. (16). As shown in figure 4, we defined the experimentally measured density by:

$$
\delta_{m}\left(F_{p}\right)=\frac{N\left(F_{p}\right)}{n\left(F_{p}\right) \cdot S_{e q \cdot g}}
$$

This permits to express easily the measured density as a function of the true damage density $\mathrm{D}(\mathrm{F})$ :

$$
\delta_{m}\left(F_{p}\right)=\int_{0}^{F_{p}} \frac{D(F)}{F} \cdot d F
$$

Thus $\mathrm{D}(\mathrm{F})$ can be easily expressed as the logarithmic derivative of the measured density $\delta_{\mathrm{m}}$.

$$
D(f)=f \cdot \frac{d \delta_{m}(f)}{d f}
$$

Since the experimental curve of $\delta_{\mathrm{m}}\left(\mathrm{F}_{\mathrm{p}}\right)$ is rather dispersed, it is better to use a functional approximation of $\delta_{\mathrm{m}}\left(\mathrm{F}_{\mathrm{p}}\right)$ to derive $\mathrm{D}(\mathrm{F})$. At high fluences (above $\mathrm{F}_{\text {cut }}$ ), damage density is well fitted by a fluence power law:

$$
\delta_{m}\left(F_{p}\right)=\alpha \cdot\left(F_{p}\right)^{\beta}
$$

$\alpha$ and $\beta$ can be calculated from the best fit of the measurements. From eq. (20) and (21), one obtains the absolute damage density:

$$
D(f)=\beta \cdot \delta_{m}(f)=\beta \cdot \frac{N(f)}{n(f) \cdot S_{\text {eq.g }}}
$$

Practically, above $\mathrm{F}_{\text {cut, }}$ to determine the absolute damage density for each fluence group, we have to know the number of damage sites and Gaussian effective area from the experiment, and then we have to determine the exponent $\beta$ from power law fitting of experimental data. 


\subsubsection{At low damage fluences, below $F_{\text {cut }}$}

The measured damage density $\delta_{\mathrm{m}}\left(\mathrm{F}_{\mathrm{p}}\right)$ is nearly constant for fluences lower than $\mathrm{F}_{\text {cut }}$. This plateau must be treated differently from the high fluence part. First of all, in this fluence range, and especially when damage sites are attributed to low fluence shots, $F_{p}$ is not a local fluence maximum. Since $\delta_{\mathrm{m}}\left(\mathrm{F}_{\mathrm{p}}\right)$ shows a very weak variation with $\mathrm{F}_{\mathrm{p}}$, it is reasonable to assume that damage density $\mathrm{D}(\mathrm{F})$ is really a constant on this fluence range. Thus $\mathrm{D}(\mathrm{F})$ is simply obtained by taking the ratio of the total number of damage sites in this fluence range, by the total area covered by these shots (with $\mathrm{F}_{\mathrm{p}}<\mathrm{F}_{\mathrm{cut}}$ ). This area is proportional to the number of these shots.

$$
D\left(F<F_{\text {cut }}\right)=\frac{N\left(F<F_{\text {cut }}\right)}{S\left(F<F_{\text {cut }}\right)}=\frac{N\left(F<F_{\text {cut }}\right)}{\frac{n\left(F<F_{\text {cut }}\right)}{n(\text { total })} \cdot S}
$$

Damage density $\mathrm{D}$ is then once again almost $\delta_{\mathrm{m}}$ multiplied by a numerical factor $\frac{n(\text { total }) . S_{\text {eq.g }}}{S}$, which is not far from 1 . The result of this treatment can be visualized in figure 4 . Since fluence is low, the number of damage sites is low too, and the result is dominated by the error bar, as we are going to see in paragraph 5.2.

\section{Error bars}

\subsection{Fluence error}

Synthesis of error margins is reported in Table 1. The first error (contributor 1) is due to the acquisition of the energy with the pyroelectric cell. This measurement is systematically compared with an absolute calorimeter calibrated yearly: the measurement uncertainty is given at $2 \%$ at $1 \sigma$ (contributor 1$)$.

A special attention has to be paid to the "qualification" of the camera used on each facility. For example series of 1000 shots at different positions around the waist position are realized in order to measure the mean equivalent area. It appears that two identical cameras (same model) gave similar results within $4 \%$ (contributor 2). The same deviation is found between three different cameras of different providers (two being 8-bit analog cameras with rectangular pixels $\left(13^{*} 11.5 \mu \mathrm{m}^{2}\right)$, the third one being a 12-bit digital camera with square pixels $\left(9.9^{*} 9.9 \mu \mathrm{m}^{2}\right)$ ). The fluctuations of cameras parameters (amplification, transmission factors) and the laser instability must be taken into account; they certainly play a part in these deviations. Thus, the different cameras give a rather similar result. These verifications were completed by checking that the effective area determined on the equivalent plane was the same as on the sample. It can be done by measuring around the waist position the areas on the two paths "camera" and "sample" (Fig. 5). For the latter, a camera is placed instead of the sample. Series of 1000 shots can then been realized for each position, each camera recording spatial profiles at the same time. It appears, in Fig. 5, a good correspondence between the two paths. From analysis, in the vicinity of the Rayleigh length, a deviation between the two paths of less than $4 \%$ was obtained (contributor 3 ). This includes the deviation coming from the cameras. It appears, in the same figure, that the deviation and fluctuations shot-to-shot are drastically reduced close to the waist position. Last, it is also important to check that the same fluctuations are recorded on the two paths. A good 
correlation between the two sets of data (of 1000 shots each) is found with a relative variation of about $6 \%$ at $1 \sigma$, contributor 4 (see Fig. 4 of ref (Lamaignère et al., 2010 )).

After reducing the total uncertainty on equivalent area measurement, a comparison between the total energy integrated on the CCD camera and the measurement from the pyroelectric cell has to be performed. The two signals should be proportional. The comparison gives a random error of about 5\% (due to the determination of the zero level on the camera, determined at each shot and repeatability of the pyroelectric cell), contributor 5. Thus, considering the whole analysis of error measurements (the different contributors are summed up in Table I with the hypothesis that there are not correlated), it is appropriate to consider for the different facilities, that the absolute fluence values are known with an accuracy around $10 \%$.

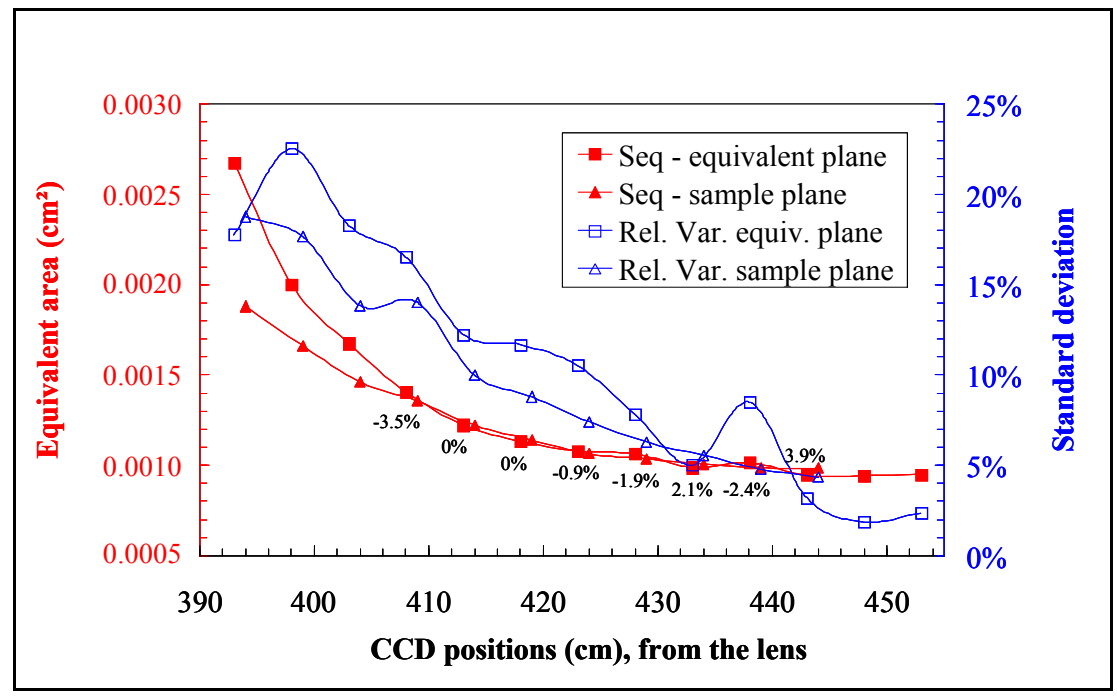

Fig. 5. Optical paths comparison. Measurements of the beam equivalent areas (full symbols) at the sample and equivalent planes with the same camera, at several positions close to the focal point. At each position, the deviations between the two optical paths are indicated.

\begin{tabular}{|c|c|c|}
\hline Contributor & Error bar at $1 \sigma(\%)$ \\
\hline 1 & Calorimeter & 2 \\
\hline 2 & Deviation between cameras & 4 \\
\hline 3 & Deviation between optical paths & 4 \\
\hline 4 & Shot-to-shot correlation between optical paths & 6 \\
\hline 5 & Deviation between camera and pyroelectric cell & 5 \\
\hline
\end{tabular}

Table 1. Synthesis of error margins for identified contributors [Error budget]. A quadratic summation provides an accuracy around $10 \%$ for the determination of fluences. 


\subsection{Intervals of confidence}

Unlike the measure of fluence, values of damage densities are rarely presented with any notion of accuracy. However, it is not possible to compare measures in one's lab or with other installations without error bars. These error bars are especially needed in the low fluence range were rare damage events are observed. The uncertainty is not on the measurement of this particular sample, because this one is now destroyed. The interesting statistical figure is the uncertainty that makes possible the comparison of two similar but physically distinct samples.

To determine the statistical error on the measurement of $\mathrm{D}(\mathrm{F})$, one assumption on the nature of the distribution of damaging defects is made: it is supposed that defects are randomly distributed over the area. A specificity of this hypothesis is that there is no interaction between defects. This supposition is common in laser damage research, and it probably holds for optical components tested with millimeter sized beams. However, it is possible to make the hypothesis that defects collaborate in damage, and draw some useful conclusions, for the case of optical multilayers or that of bulk damage of KDP crystals.

The assumption of randomly distributed and independent defects is applied to any set of defects: For example a set of defects damaging at a given fluence is supposed to be distributed that way in the sample. So a set of defects is damaging in a given fluence range. A damage test is an experimental sampling of a distribution of defects that characterizes a very large area, for example the area of all the optical components and samples produced with the same recipes. The error made will be calculated as a possible discrepancy between density obtained at the sample and the "true" density that would be measured if the whole production was damage tested.

The uncertainty depends on the number of damage sites generated within each fluence group. By considering a Poisson distribution, it is rather straightforward to determine the interval of possible measurements when the true characteristic is known. Let us define:

$\Sigma$ : The surface area of the total production

$\mathrm{N}$ : number of potential damage sites on the total production

$\mathrm{k}$ : number of detected damage sites on $\mathrm{S}$

$v=\mathrm{N} \mathrm{S} / \Sigma$, number of expected damage sites on $S$

$\mathrm{k}$ is not equal to $\mathrm{v}$, but rather follows Poisson law, when $\Sigma$ is very large compared to $S$, the area of the tested surface. Thus the probability of finding a given $\mathrm{k}$ value is:

$$
P_{v}(k)=\frac{v^{k} e^{-v}}{k !}
$$

This formula (24) is only the first step. The problem is then inversed since we want to know $v$ from the measurement $\mathrm{k}$. The main difficulty lies in the fact that we are interested in small values of $\mathrm{k}($ or $v)$. When a law of probability is determined with a high enough number of events, then one can use the law of large numbers to express neatly the error in terms of erf function. In case of rare events, especially in the low fluence range, we had to make a special derivation (the full demonstration is given in reference (Lamaignère et al., 2007)).

Let $\mathrm{k}$ be the known number of detected damage sites. The interval of values of $v$, for the confidence to be better than 1- $\varepsilon$ can be written: 
with

$$
v \in\left[v_{\min } ; v_{\max }\right]
$$

and

$$
\int_{v=0}^{v_{\min }} \frac{v^{k} e^{-v}}{k !} \cdot d v=\frac{\varepsilon}{2} \text { when } \mathrm{k} \neq 0 \quad ; \quad v_{\min }=0 \text { when } \mathrm{k}=0
$$

$$
\int_{v=v_{\max }}^{+\infty} \frac{v^{k} e^{-v}}{k !} \cdot d v=\frac{\varepsilon}{2}
$$

This means that we calculate a probability 1- $\varepsilon$ for $v$ to lie in the interval between $v_{\min }$ and $v_{\max }$. In this section, we know use specifically the confidence limits that corresponds with 2 standard deviation $(2 \sigma)$ of a Gaussian variable $\varepsilon=.0455$, or $\varepsilon / 2=.02275$.

The confidence limits are very far apart when the measured number of damage sites is low. Table 2 gives a numerical derivation of these limits for low $\mathrm{k}$ values. At $\mathrm{k}=0$, when no damage site is detected, we can only say that the average number of sites is lower than 3.7 with an error rate of $2.3 \%$. This number of sites must be translated into a density.

One should notice that these error bars are only given by the statistical variations due to the limited number of data (connected to the size of the sample). Potential errors due to inaccurate damage detection are not taken into account.

\begin{tabular}{|c|c|c|c|c|c|c|c|c|c|c|c|c|c|c|c|c|c|c|c|c|c|}
\hline $\mathbf{k}$ & $\mathbf{0}$ & $\mathbf{1}$ & $\mathbf{2}$ & $\mathbf{3}$ & $\mathbf{4}$ & $\mathbf{5}$ & $\mathbf{6}$ & $\mathbf{7}$ & $\mathbf{8}$ & $\mathbf{9}$ & $\mathbf{1 0}$ & $\mathbf{1 1}$ & $\mathbf{1 2}$ & $\mathbf{1 3}$ & $\mathbf{1 4}$ & $\mathbf{1 5}$ & $\mathbf{1 6}$ & $\mathbf{1 7}$ & $\mathbf{1 8}$ & $\mathbf{1 9}$ & $\mathbf{2 0}$ \\
\hline$v_{\min }$ & 0 & 0,2 & 0,6 & 1,1 & 1,6 & 2,2 & 2,8 & 3,4 & 4 & 4,7 & 5,4 & 6,1 & 6,8 & 7,6 & 8,3 & 9 & 9,8 & 11 & 11 & 12 & 13 \\
\hline$v_{\max }$ & 3,7 & 5,6 & 7,2 & 8,8 & 10 & 12 & 13 & 15 & 16 & 17 & 19 & 20 & 21 & 22 & 24 & 25 & 26 & 27 & 29 & 30 & 31 \\
\hline
\end{tabular}

Table 2. Interval of confidence of $v$ for a given measured value of $\mathrm{k}$.

\section{Results}

This chapter is dedicated to experimental results and illustrates:

- The complementarity of the several procedures which can be indifferently used depending on the information asked;

- The repeatability;

- $\quad$ The reproducibility; these two notions permit to validate the whole procedures;

- The representativeness of tests realized with small beams compared to large and real beams on high laser facilities.

\subsection{Complementarity}

Figure 6 shows results obtained on the same optical component tested with the 1/1 and rasterscan procedures, and on the same facility. Results are directly reported in terms of damage density with the presented formalism in $\$ 4.1$ and 4.2. During rasterscan, about 6000 shots (this corresponds to a scanned area of about $6 \mathrm{~cm}^{2}$ ) are fired with fluences between 2.5 and $4.5 \mathrm{~J} / \mathrm{cm}^{2}$. During the $1 / 1$ tests, only 200 shots have been fired with fluences between 4.5 and $6.5 \mathrm{~J} / \mathrm{cm}^{2}$. These results, which were presented, in the past, in terms of damage probabilities, are translated in terms of damage densities. The good complementarity of the two test results leads to validate the developed formalisms (for clarity, fluence error bars are 
not reported). We remark that due to a large number of tested sites, low damage densities are available with the rasterscan technique. On the opposite, 1/1 test covers damage probabilities between 0 and 1 , and due to the small illuminated area, damage densities available are higher. We observe that the intervals of confidence are smaller for rasterscan procedures, due to a large number of damage sites in spite of small damage densities, than for $1 / 1$ where a limited number of sites are tested. Nevertheless, the overlap of damage densities indicates a good measurement complement and that data reduction permits to determine repeatable damage densities; in spite of a non $100 \%$ overlap during rasterscan and a small number of tested sites during $1 / 1$. Identical results were also obtained with different pulse durations and spatial beam profiles.

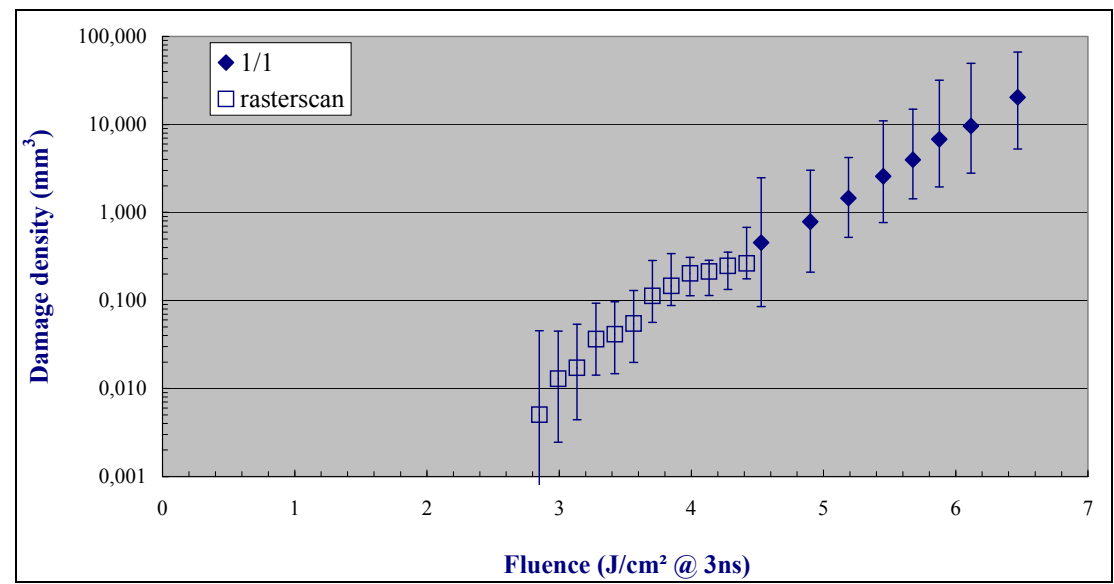

Fig. 6. Comparison of $1 / 1$ and rasterscan procedures. Damage densities vs fluence measured on the same optical component with the $1 / 1$ test procedure (diamonds) and 3 rasterscans at 3 different fluences (squares).

\subsection{Repeatability}

It is necessary to check that the procedures applied on similar optics give always similar results on a unique set-up. This should be done regularly. Fig. 7 shows results obtained on 3 samples from the same vendor, tested on the same facility on a one year period. Damage densities lay inside confidence limits. This result and those obtained with other lasers (not presented here) show that it is possible to achieve a good repeatability.

\subsection{Beam overlap}

A non-negligible parameter of the procedure is the shot-to-shot step i.e. the beam overlap. In order to scan a large area and to be sure to irradiate all the defects, a good overlap is necessary. That is possible with top-hat beams but not with Gaussian ones. In the latter case, it is preferable not to use too small a step, in order to avoid that the defects experience a long irradiation ramp, that the scan duration is too long and that damage grows due to successive shots on the same site. Moreover, a good correspondence between damage and fluence maps requires the step not to be too small. On the contrary, a large step implies a large area to be scanned or a low statistic. At last, whatever steps and overlaps, data 


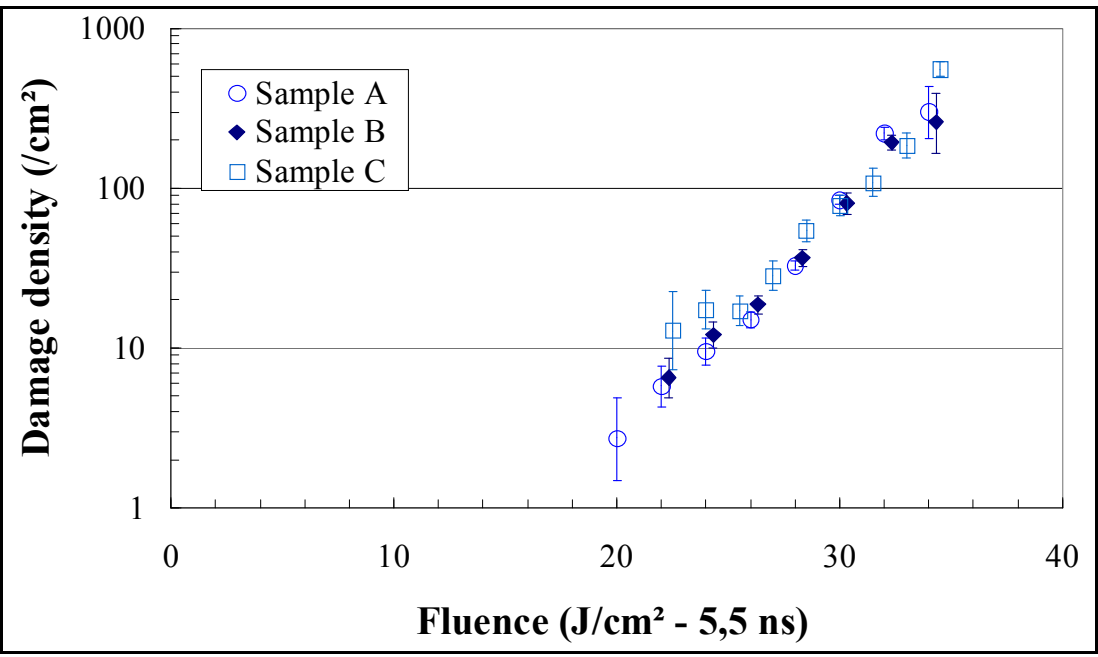

Fig. 7. Repeatability. Damage densities D vs fluence F for three different optical components (from the same vendor) tested on the same facility within a one year period with the rasterscan procedure.

treatment must provide the same and accurate results. In Fig. 8 results obtained on the same optic $\left(330 * 330 \mathrm{~mm}^{2}\right)$ for 5 different steps are reported $(0.15 ; 0.3 ; 0.6 ; 1 ; 2 \mathrm{~mm}$ corresponding to an overlap of $95 ; 92 ; 66 ; 31$ and $3 \%$ ). For a step smaller than $0.15 \mathrm{~mm}$, catastrophic damage growth was observed. No tests were conducted under this value. As can be seen in

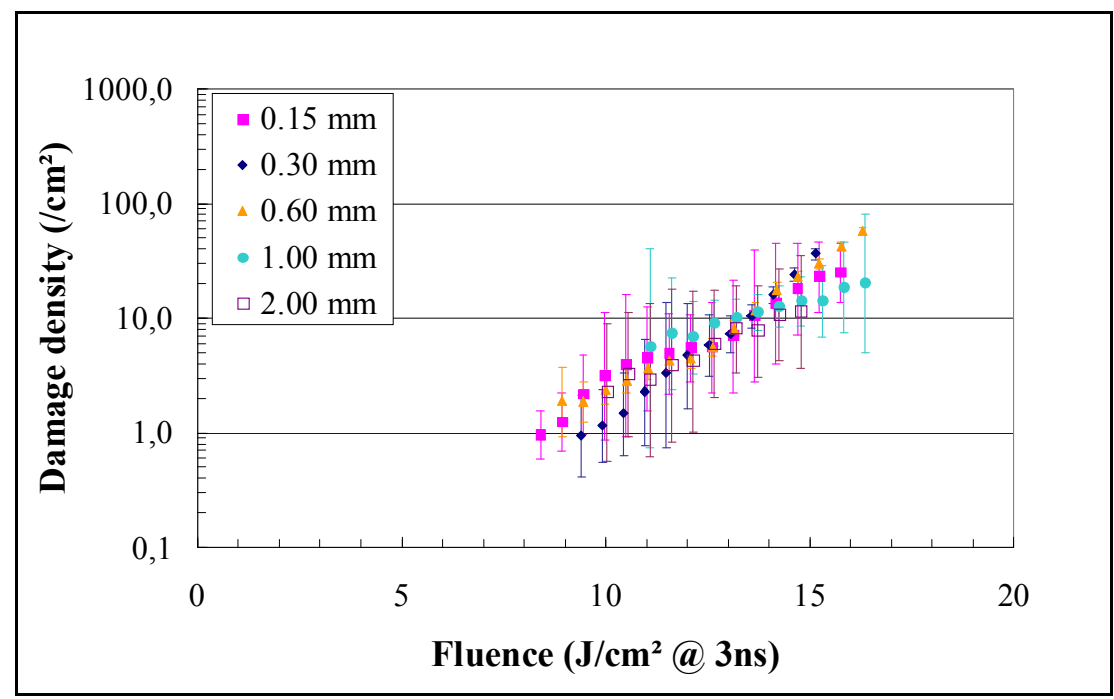

Fig. 8. Steps. Damage densities vs fluence $D(F)$ for the same optic tested on the same facility with 5 different steps on 5 different zones. Data have been processed following data reduction presented in $\S 4.2$, this example is extracted from a Gaussian test. 
Fig. 8, from 9 to $15 \mathrm{~J} / \mathrm{cm}^{2}$, results are gathered inside the largest interval of confidence (given at $2 \sigma$ ). These results indicate that this procedure, with its data treatment, is able to provide comparable measurements for a wide range of beam overlap. Consequently, comparison is possible between several facilities where the shot-to-shot step inevitably varies from one test to another.

\subsection{Reproducibility}

The comparison is on 4 optical components from the same batch. Due to the limited available area on each sample, data exploration is realized for fluences between 10 and 20 $\mathrm{J} / \mathrm{cm}^{2}$. In this range, damage density increases quickly with fluence and values are sufficiently high for the number of damage sites to be high enough. For lower fluences and lower damage densities, the areas to be scanned become too large and the error bars could be too high to make any conclusion. For each sample, an area about $40 \mathrm{~cm}^{2}$ was irradiated.

Data treatment presented in $\S 4.2$ is first applied (see insert of Fig. 9). Next pulselength scaling is used. The best correspondence is obtained when $\alpha$ is equal to 0.6. (Fig. 9), value slightly different from the usual scaling $\tau^{1 / 2}$. Up to $18 \mathrm{~J} / \mathrm{cm}^{2}$, measurements are gathered inside the largest interval of confidence (given at $2 \sigma$ ). Fluence error bars are not reported for clarity (values are provided with an accuracy around 10\%) but taking into account both fluence error bars and level of confidence, results are quite comparable. Above a few hundred damage per $\mathrm{cm}^{2}$, damage sites can aggregate and the comparison is no more feasible.

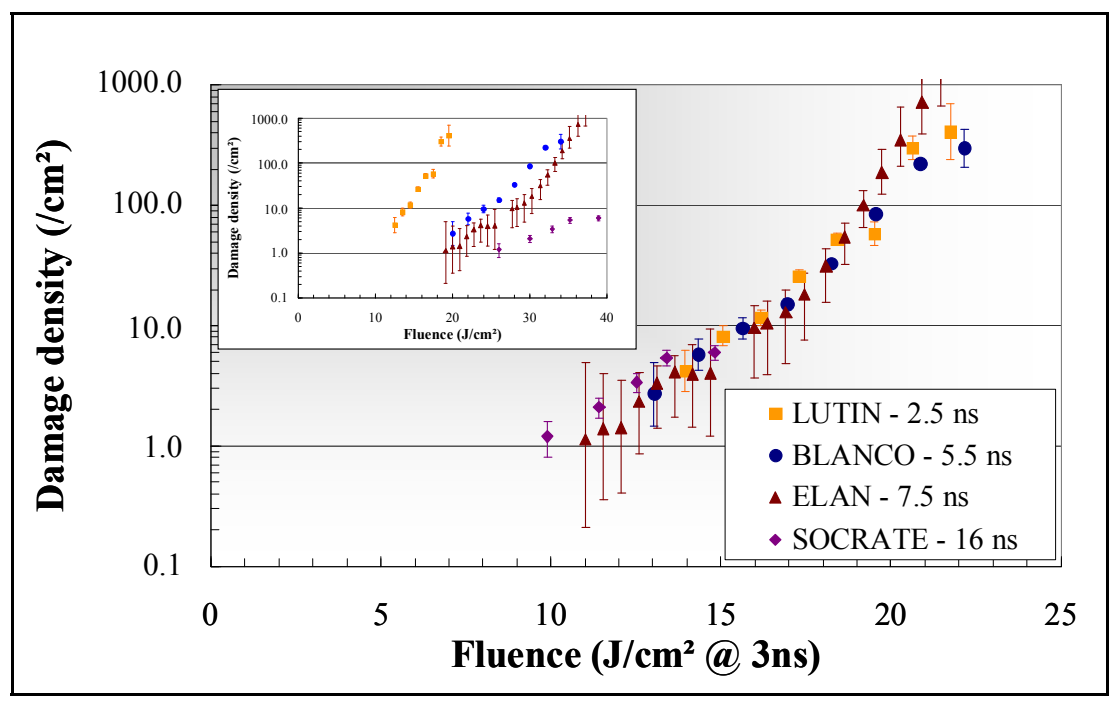

Fig. 9. Reproducibility. Damage densities vs fluence for several components (with the same polishing process) tested on 4 different facilities. Fluences are scaled using a temporal scaling law $\left(\tau^{\alpha}\right)$ with an $\alpha$ exponent of 0.6 . In the insert, the raw data are reported. 


\subsection{Representativeness}

For the question of representatives, an area of $40 \mathrm{~cm}^{2}$ has been tested on a large optic with the small beam rasterscan procedure, i.e. illumination, damage detection and data processing. A second optics has been tested with the large beam. For this comparison, the number of parameters, that are different, is reduced selecting shots that are quite similar: the pulses are single mode longitudinally; the temporal profiles are Gaussian with equivalent pulse durations $\tau=2.5$ and $2.3 \mathrm{~ns}$, respectively. Data treatments are first applied. Next pulselength scaling is applied with the help of a temporal scaling law $\left(F \sim \tau^{\alpha}\right)$ and $\alpha=0.6$, this value being previously determined (see \$6.4). Nevertheless, the two pulse durations being close, the comparison can also be made with the usual scaling $\tau^{2 / 2}$ or without the use of any scaling law. The absolute fluence values are known with an accuracy of about $10 \%$ (Lamaignère et al., 2010); the sources of errors on the two facilities being different, it is compulsory to take into account these errors. Figure 10 shows that measurements are gathered inside the largest interval of confidence (given at $2 \sigma$ ) and thus the two results are quite comparable. This confirms the reproducibility that was noted in the previous paragraph.

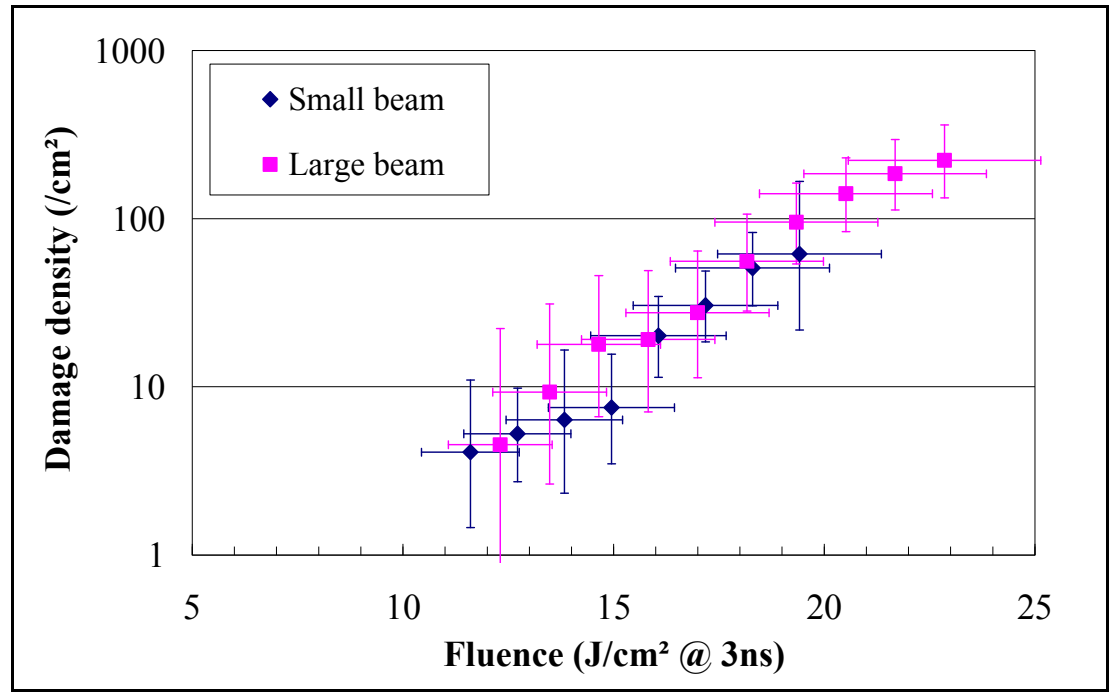

Fig. 10. Damage densities versus fluence measured on two optics from the same batch with small and large beams.

\section{Conclusion}

With rigorous data analysis and treatment, it is possible to measure damage density on an optical component with high accuracy and repeatability, whatever the beam overlap and the beam shape. Since tests are destructive, the same area cannot be measured on two different instruments. However the consideration of error bars on damage density allows comparing results from several components assumed to be comparable. 
A particular attention has to be devoted to the error budget on fluence determination, more precisely on the measurement of beam equivalent area. CCD cameras have to be carefully qualified. Thus the error on calculation could be estimated. It is vital to ensure that this equivalent area determined on the reference path is equal to that on the sample controlling the CCD position and by verifying that the optics in front of the camera do not alter the beam profile. This measurement has to be recorded at the frequency laser to monitor shotto-shot laser fluctuations. The calculations on error bars not only allow comparing results from several samples tested on several facilities but also give an upper limit of damage density, particularly useful when a small area is scanned or in a low damage density range.

Depending on the available area on the sample to be tested and the level of damage density, it appears that the $1 / 1$ and rasterscan procedures are comparable and complementary with the use of an appropriate data reduction. More, these procedures give access to representative measurements when compared to results and behaviours observed on high laser facilities irradiated with very large beams.

\section{References}

Bercegol, H., Boscheron, A., DiNicola, J.M., Journot, E., Lamaignère, L., Néauport J. \& Razé, G. (2008). Laser damage phenomena relevant to the design and operation of an ICF laser driver, J. Phys. Conf. Ser., 112, 032013-032016.

DeMange, P., Carr, C.W., Radousky, H.B., \& Demos, S.G. (2004). System for evaluation of laser-induced damage performance of optical materials for large aperture lasers, Rev. Sci. Instr. 75, 3298.

ISO standards: (ISO 21254-1:2011) - (ISO 21254-2:2011) - (ISO 21254-3:2011).

Feit, M.D., Rubenchik, A.M., Kozlowski, M.R., Génin, F.Y., Schwartz, S. \& Sheehan, L.M. (1999). Extrapolation of damage test data to predict performance of large-area NIF optics at $355 \mathrm{~nm}$, Proc. SPIE 3578, 226-234.

Lamaignère, L., Bouillet, S., Courchinoux, R., Donval, T., Josse, M., Poncetta, J.C. \& Bercegol, H. (2007). An accurate, repeatable, and well characterized measurement of laser damage density of optical materials, Rev. Sci. Instr. 78, 103105.

Lamaignère, L., Donval, T., Loiseau, M., Poncetta, J.C., Raze, G., Meslin, C., Bertussi, B. \& Bercegol, H. (2009). Accurate measurements of laser-induced bulk damage density, Meas. Sci. Technol. 20, 095701.

Lamaignère, L., Balas, M., Courchinoux, R., Donval, T., Poncetta, J.C., Reyné, S., Bertussi, B. \& Bercegol, H. (2010). Parametric study of laser-induced surface damage density measurements: Toward reproducibility, J. Appl. Phys. 107, 023105.

Lamaignère, L., Dupuy, G., Donval, T., Grua, P. \& Bercegol, H. (2011). Comparison of laserinduced surface damage density measurements with small and large beams: toward representativness, Applied Optics 50, 442.

Negres, R.A., Norton, M.A., Cross, D.A. \& Carr, C.W., (2010). Growth behavior of laserinduced damage on fused silica optics under UV, ns laser irradiation, Opt. Express 18, 19966-19976.

Norton, M.A., Donohue, E.E., Feit, M.D., Hackel, R.P., Hollingsworth, W.G., Rubenchik, A.M. \& Spaeth, M.L. Growth of laser damage in $\mathrm{SiO}_{2}$ under multiple wavelength irradiation, Proc.SPIE 5991, 599108 (2006). 


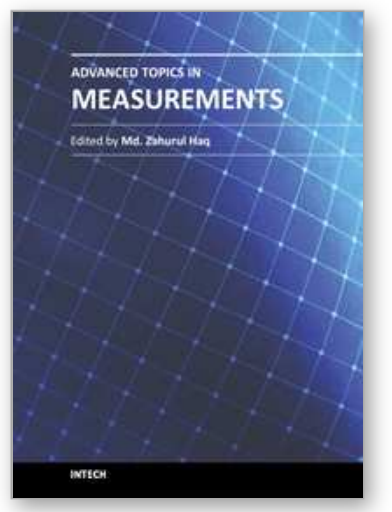

\author{
Advanced Topics in Measurements \\ Edited by Prof. Zahurul Haq
}

ISBN 978-953-51-0128-4

Hard cover, 400 pages

Publisher InTech

Published online 07, March, 2012

Published in print edition March, 2012

Measurement is a multidisciplinary experimental science. Measurement systems synergistically blend science, engineering and statistical methods to provide fundamental data for research, design and development, control of processes and operations, and facilitate safe and economic performance of systems. In recent years, measuring techniques have expanded rapidly and gained maturity, through extensive research activities and hardware advancements. With individual chapters authored by eminent professionals in their respective topics, Advanced Topics in Measurements attempts to provide a comprehensive presentation and in-depth guidance on some of the key applied and advanced topics in measurements for scientists, engineers and educators.

\title{
How to reference
}

In order to correctly reference this scholarly work, feel free to copy and paste the following:

Laurent Lamaignère (2012). Laser-Induced Damage Density Measurements of Optical Materials, Advanced Topics in Measurements, Prof. Zahurul Haq (Ed.), ISBN: 978-953-51-0128-4, InTech, Available from: http://www.intechopen.com/books/advanced-topics-in-measurements/laser-induced-damage-densitymeasurements-of-optical-materials-

\section{INTECH}

open science | open minds

\section{InTech Europe}

University Campus STeP Ri

Slavka Krautzeka 83/A

51000 Rijeka, Croatia

Phone: +385 (51) 770447

Fax: +385 (51) 686166

www.intechopen.com

\section{InTech China}

Unit 405, Office Block, Hotel Equatorial Shanghai

No.65, Yan An Road (West), Shanghai, 200040, China

中国上海市延安西路65号上海国际贵都大饭店办公楼 405 单元

Phone: +86-21-62489820

Fax: +86-21-62489821 
(C) 2012 The Author(s). Licensee IntechOpen. This is an open access article distributed under the terms of the Creative Commons Attribution 3.0 License, which permits unrestricted use, distribution, and reproduction in any medium, provided the original work is properly cited. 